\title{
Free-surface Wave Interaction with a Thick Flexible Dock or Very Large Floating Platform
}

\author{
A.J. Hermans*
}

\begin{abstract}
In this paper the recently developed semi-analytic method to solve the free-surface wave interaction with a thin elastic plate is extended to the case of a plate of finite thickness. The method used is based on the reformulation of the differential-integral equation for this problem. The thickness of the plate is chosen such that the elastic behavior of the plate can be described by means of thin plate theory, while the water pressure at the plate is applied at finite depth. The water depth is finite.
\end{abstract}

\section{Introduction}

We consider the two-dimensional interaction of an incident wave with a flexible floating dock or very large floating platform (VLFP) with finite draft. The water depth is finite. The case of a rigid dock is a classical problem. For instance Mei and Black [1] have solved the rigid problem, by means of a variational approach. They considered a fixed bottom and fixed free surface obstacle, so they also covered the case of small draft. After splitting the problem in a symmetric and an antisymmetric one the method consists of matching of eigenfunction expansions of the velocity potential and its normal derivative at the boundaries of two regions. In principle, their method can be extended to the flexible platform case. Recently we derived a simpler method for both the moving rigid and the flexible dock [2]. However we considered objects with zero draft only. In this paper we extend our approach to the case of finite, but small, draft. The draft is small compared to the length of the platform to be sure that we may use as a model, for the elastic plate, the thin plate theory, while the water pressure at the plate is applied at finite depth. The method is based on a direct application of Green's theorem, combined with an appropriate choice of expansion functions for the potential in the fluid region outside the platform and the deflection of the plate. The integral equation obtained by the Green's theorem is transformed into an integral-differential equation by making use of the equation for the elastic plate deflection. One must be careful in choosing the appropriate Green's function. It is crucial to use a formulation of the Green's function consisting of an integral expression only. In Appendix A we derive such a Green's function for the twodimensional case. One may derive an expression as can be found in the article of Wehausen and Laitone [3] after application of Cauchy's residue lemma. In the three dimensional case one also may derive such an expression. The advantage of this version of the source function is that one may work out the integration with respect to the space coordinate first and apply the residue lemma afterwards. In the case of a zero draft platform this approach resulted in the dispersion relation in the plate region and an algebraic set of equations for the coefficients of the deflection only. Here we derive a coupled algebraic set of equations for the expansion coefficients of the potential in the fluid region and the deflection.

\section{Mathematical formulation for the finite draft problem}

In this section we derive the general formulation for the diffraction of waves by a flexible platform of general geometric form. The fluid is ideal, so we introduce a velocity potential using $\mathbf{V}(\mathbf{x}, t)=$

${ }^{*} T U$ Delft, Faculty of Electrical Engineering, Mathematics and Computer Science, Department of Applied Mathematical Analysis, Mekelweg 4, 2628 CDDelft, The Netherlands, e-mail a.j.hermans@ewi.tudelft.nl 
$\nabla \Phi(\mathbf{x}, t)$, where $\mathbf{V}(\mathbf{x}, t)$ is the fluid velocity vector. Hence $\Phi(\mathbf{x}, t)$ is a solution of the Laplace equation

$$
\Delta \Phi=0 \quad \text { in the fluid, }
$$

together with the linearized kinematic condition, $\Phi_{z}=\tilde{w}_{t}$, and dynamic condition, $p / \rho=-\Phi_{t}-$ $g \tilde{w}$, at the mean water surface $z=0$, where $\tilde{w}(x, y, t)$ denotes the free surface elevation, and $\rho$ is the density of the water. The linearized free surface condition outside the platform, $z=0$ and $(x, y) \in \mathcal{F}$, becomes:

$$
\frac{\partial^{2} \Phi}{\partial t^{2}}+g \frac{\partial \Phi}{\partial z}=0
$$

The platform is situated at the mean free-surface $z=0$, its thickness is $d$. The platform is modelled as an elastic plate with zero thickness. The neutral axis of the plate is at $z=0$, while the water pressure distribution is applied at $z=-d$. Meylan et al [4] have considered finite thickness as well. They consider the elastic equation for the deflection of a plate of finite thickness, however they apply the equation of motion at $z=0$. They show for large platforms a minor influence due to the change of the elastic model. Our elastic model can easily modified by changing the fourth order differential operator, but due to lack of knowledge of suitable parameters we decided not to do so. So we neglect horizontal and torsional motion. To describe the vertical deflection $\tilde{w}(x, y, t)$, we apply the isotropic thin-plate theory, which leads to an equation for $\tilde{w}$ of the form

$$
m(x, y) \frac{\partial^{2} \tilde{w}}{\partial t^{2}}=-\left(\frac{\partial^{2}}{\partial x^{2}}+\frac{\partial^{2}}{\partial y^{2}}\right)\left(D(x, y)\left(\frac{\partial^{2} \tilde{w}}{\partial x^{2}}+\frac{\partial^{2} \tilde{w}}{\partial y^{2}}\right)\right)+\left.p\right|_{z=-d}
$$

where $m(x, y)$ is the piece-wise constant mass of unit area of the platform while the piece-wise constant $D(x, y)$ is its equivalent flexural rigidity. We differentiate (3) with respect to $t$ and use the kinematic and dynamic condition to arrive at the following equation for $\Phi$ at $z=-d$ in the platform area $(x, y) \in \mathcal{P}$ :

$$
\left\{\left(\frac{\partial^{2}}{\partial x^{2}}+\frac{\partial^{2}}{\partial y^{2}}\right)\left(\frac{D(x, y)}{\rho g}\left(\frac{\partial^{2}}{\partial x^{2}}+\frac{\partial^{2}}{\partial y^{2}}\right)\right)+\frac{m(x, y)}{\rho g} \frac{\partial^{2}}{\partial t^{2}}+1\right\} \frac{\partial \Phi}{\partial z}+\frac{1}{g} \frac{\partial^{2} \Phi}{\partial t^{2}}=0 .
$$

Due to the fact that the plate is freely floating we do not consider the hydrostatic pressure.

The edges of the platform are free of shear forces and moment. We assume that the flexural rigidity is constant along the edge and its derivative normal to the edge equals zero. Also, we assume that the radius of curvature, in the horizontal plane, of the edge is large. Hence, the edge may be considered to be straight locally. We then have the following boundary conditions at the edge:

$$
\frac{\partial^{2} \tilde{w}}{\partial n^{2}}+v \frac{\partial^{2} \tilde{w}}{\partial s^{2}}=0 \text { and } \frac{\partial^{3} \tilde{w}}{\partial n^{3}}+(2-v) \frac{\partial^{3} \tilde{w}}{\partial n \partial s^{2}}=0
$$

where $v$ is Poisson's ratio, $n$ is in the normal direction, in the horizontal plane, along the edge and $s$ denotes the arc-length along the edge. At the bottom of the fluid region $z=-h$ we have:

$$
\frac{\partial \Phi}{\partial z}=0
$$

We assume that the velocity potential is a time-harmonic wave function, $\Phi(\mathbf{x}, t)=\phi(\mathbf{x}) \mathrm{e}^{-\mathrm{i} \omega t}$. We introduce the following parameters:

$$
K=\frac{\omega^{2}}{g}, \mu=\frac{m \omega^{2}}{\rho g}, \mathcal{D}=\frac{D}{\rho g} .
$$

In a practical situation the total length $L$ of the platform is a few thousand meters. We obtain at the free surface, $z=0$

$$
\frac{\partial \phi}{\partial z}-K \phi=0
$$

and at the plate, $z=-d$, for a single strip,

$$
\left\{\mathcal{D}\left(\frac{\partial^{2}}{\partial x^{2}}+\frac{\partial^{2}}{\partial y^{2}}\right)^{2}-\mu+1\right\} \frac{\partial \phi}{\partial z}-K \phi=0 .
$$


The potential of the undisturbed incident wave is given by:

$$
\phi^{\text {inc }}(\mathbf{x})=\frac{g \zeta_{\infty}}{\mathrm{i} \omega} \frac{\cosh \left(k_{0}(z+h)\right)}{\cosh \left(k_{0} h\right)} \exp \left\{\mathrm{i} k_{0}(x \cos \beta+y \sin \beta)\right\}
$$

where $\zeta_{\infty}$ is the wave amplitude in the original coordinate system, $\omega$ the frequency, while the wave number $k_{0}$ is the positive real solution of the dispersion relation,

$$
k_{0} \tanh \left(k_{0} h\right)=K,
$$

for finite water depth. We restrict ourselves to the case of normal incidence, $\beta=0$. In [6]it is shown that the extension to oblique waves can be done easily.

To obtain an integral equation for the deflection $\tilde{w}(x, y, t)=\mathfrak{R}\left[w(x, y) \mathrm{e}^{-i \omega t}\right]$ of the platform, see [5] and [6], it is very convenient to apply the Green's theorem, making use of the Green's function, $\mathcal{G}(\mathbf{x} ; \boldsymbol{\xi})$, that fulfills boundary conditions at the seabed (6) and at the free surface (7). Application of Green's theorem in the fluid domain leads to the following expression for the potential function,

$$
4 \pi \phi(\mathbf{x})=4 \pi \phi^{i n c}(\mathbf{x})+\int_{C} \phi(\boldsymbol{\xi}) \frac{\partial \mathcal{G}(\mathbf{x}, \boldsymbol{\xi})}{\partial n} \mathrm{~d} S+\int_{P}\left(\phi(\boldsymbol{\xi}) \frac{\partial \mathcal{G}(\mathbf{x}, \boldsymbol{\xi})}{\partial \zeta}-\frac{\partial \phi(\boldsymbol{\xi})}{\partial \zeta} \mathcal{G}(\mathbf{x} ; \boldsymbol{\xi})\right) \mathrm{d} S .
$$

The first integral is along the vertical sides of the platform, where the normal velocity of the fluid equals zero. The second integral is along the flat bottom. In the two-dimensional case, $(x, z)$-plane, the expression for the total potential becomes:

$$
\begin{aligned}
2 \pi \phi(x, z)=2 \pi \phi^{\text {inc }}(x, z)+\int_{-d}^{0}\left(\phi(0, \zeta) \frac{\partial \mathcal{G}(x, z ; 0, \zeta)}{\partial \xi}-\phi(l, \zeta) \frac{\partial \mathcal{G}(x, z ; l, \zeta)}{\partial \xi}\right) \mathrm{d} \zeta \\
+\int_{0}^{l}\left(\phi(\xi,-d) \frac{\partial \mathcal{G}(x, z ; \xi,-d)}{\partial \zeta}-\frac{\partial \phi(\xi,-d)}{\partial \zeta} \mathcal{G}(x, z ; \xi,-d)\right) \mathrm{d} \xi .
\end{aligned}
$$

We continue with the two-dimensional case.

The Green's function $G(x, z ; \xi, \zeta)$ for the two dimensional case can be derived by means of a Fourier transform with respect to the $x$-coordinate. As is shown in Apendix A it has the form:

$$
G(x, z ; \xi, \zeta)=\int_{-\infty}^{\infty} \frac{1}{\gamma} \frac{K \sinh \gamma z+\gamma \cosh \gamma z}{K \cosh \gamma h-\gamma \sinh \gamma h} \cosh \gamma(\zeta+h) \mathrm{e}^{\mathrm{i} \gamma(x-\xi)} \mathrm{d} \gamma \quad \text { for } z>\zeta
$$

and

$$
G(x, z ; \xi, \zeta)=\int_{-\infty}^{\infty} \frac{1}{\gamma} \frac{K \sinh \gamma \zeta+\gamma \cosh \gamma \zeta}{K \cosh \gamma h-\gamma \sinh \gamma h} \cosh \gamma(z+h) \mathrm{e}^{\mathrm{i} \gamma(x-\xi)} \mathrm{d} \gamma \quad \text { for } z<\zeta .
$$

If we close the contour of integration in the complex $\gamma$-plane we obtain the complex version of formula (13.34), as can be found in Wehausen and Laitone [3]

$$
G(x, z ; \xi, \zeta)=-2 \pi \mathrm{i} \sum_{i=0}^{\infty} \frac{1}{k_{i}} \frac{k_{i}^{2}-K^{2}}{h k_{i}^{2}-h K^{2}+K} \cosh k_{i}(z+h) \cosh k_{i}(\zeta+h) \mathrm{e}^{\mathrm{i} k_{i}|x-\xi|},
$$

where $k_{0}$ and $k_{i}, i=1, \cdots, \infty$ are the positive real and positive imaginary zeros of the dispersion relation (10).

The advantage of this formulation for the Green's function is that, by means of the Green's theorem, we can derive the algebraic set of equations for the expansion coefficients by carrying out the integration with respect to the spatial variable analytically first.

It is well known that for the rigid case, Mei and Black [1], the potential can be expanded in eigenfunctions in the regions outside and underneath the platform. In the traditional approach continuity of mass and velocity leads to sets of equations at $x=0$ and $x=l$ respectively. The use of orthogonality relations then gives a set of equations for the unknown coefficients. In the case of zero thickness it is shown by Hermans [2] that a set of algebraic equations can be obtained for the expansion coefficients of the deflection alone. Here we also use this approach to obtain a coupled set of algebraic equations for the finite thickness case as well. It is also possible to make a non-orthogonal expansion, see for instance [7], of the potential underneath the flexible platform. In that case one can express, a posteriori, the deflection as an expansion in exponential functions. The dispersion relations derived by both approaches are the same, as expected. 


\section{Semi analytic solution}

Equation (12) and or the three-dimensional version (11), together with the condition at the bottom of the plate (8), can be solved by by means of a numerical diffraction code based on WAMIT. However, it is interesting to see how one can solve the equations semi-analytical for simple geometries. Here we work out the case of a strip.

We eliminate in relation (12) the function $\phi(\xi,-d)$ by using equation (8) and the kinematic condition

$$
\phi_{\zeta}(\xi,-d)=-\mathrm{i} \omega w(\xi) .
$$

Thus we obtain,

$$
\begin{gathered}
2 \pi \phi(x, z)=2 \pi \phi^{\mathrm{inc}}(x, z)+\int_{-d}^{0}\left(\phi(0, \zeta) \frac{\partial \mathcal{G}(x, z ; 0, \zeta)}{\partial \xi}-\phi(l, \zeta) \frac{\partial \mathcal{G}(x, z ; l, \zeta)}{\partial \xi}\right) \mathrm{d} \zeta \\
-\mathrm{i} \omega \int_{0}^{l}\left(\frac{1}{K}\left(\mathcal{D} \frac{\partial^{4}}{\partial \xi^{4}}-\mu+1\right) w(\xi) \frac{\partial \mathcal{G}(x, z ; \xi,-d)}{\partial \zeta}-w(\xi) \mathcal{G}(x, z ; \xi,-d)\right) \mathrm{d} \xi .
\end{gathered}
$$

We assume that the deflection $w(x)$ can be written as an expansion in exponential functions, truncated at $N+2$ terms of the form,

$$
w(x)=\zeta_{\infty} \sum_{n=0}^{N+1}\left(a_{n} \mathrm{e}^{\mathrm{i} \kappa_{n} x}+b_{n} \mathrm{e}^{-\mathrm{i} \kappa_{n}(x-l)}\right) .
$$

The values for $\kappa_{n}$ follow from a 'dispersion' relation, yet to be determined. If we consider $\kappa_{n}$ 's with either real positive values or, if they are complex, with positive imaginary part, then the first part of expression (18) expresses modes travelling and evanescent to the right. The second part then describes modes travelling and evanescent to the left.

Furthermore we expand the potential function for $x \leq 0$ and $x \geq l$ in series of orthogonal eigenfunctions, truncated at $N$ terms

$$
\phi(x, z)=\frac{g \zeta_{\infty}}{\mathrm{i} \omega}\left(\frac{\cosh k_{0}(z+h)}{\cosh k_{0} h} \mathrm{e}^{\mathrm{i} k_{0} x}+\sum_{n=0}^{N-1} \alpha_{n} \frac{\cosh k_{n}(z+h)}{\cosh k_{n} h} \mathrm{e}^{-\mathrm{i} k_{n} x}\right) \quad \text { for } x \leq 0
$$

and

$$
\phi(x, z)=\frac{g \zeta_{\infty}}{\mathrm{i} \omega} \sum_{n=0}^{N-1} \beta_{n} \frac{\cosh k_{n}(z+h)}{\cosh k_{n} h} \mathrm{e}^{\mathrm{i} k_{n}(x-l)} \text { for } x \geq l,
$$

The difference in the number of expansion functions in (18) is due to the fact that we have four boundary conditions at the edge of the plate (5). The coefficients $\alpha_{0}$ and $\beta_{0}$ are the reflection and transmission coefficients respectively. it should be noticed that the potential under the platform is not expanded in a set of orthogonal eigenfunctions. By the way, such a set does not exist. Extension of the solution along the bottom of the platform in the flow region is simply done by application of (17). We have introduced $4 N+4$ unknown coefficients. Next we derive an algebraic set of equations for these coefficients.

First we take $(x, z)$ at the bottom of the plate, this leads to the following equation

$$
\begin{aligned}
& 2 \pi\left(\mathcal{D} \frac{\partial^{4}}{\partial x^{4}}-\mu+1\right) w(x)= \\
& -2 \pi \frac{K}{\mathrm{i} \omega} \phi^{\text {inc }}(x,-d)-\frac{K}{\mathrm{i} \omega} \int_{-d}^{0}\left(\phi(0, \zeta) \frac{\partial \mathcal{G}(x,-d ; 0, \zeta)}{\partial \xi}-\phi(l, \zeta) \frac{\partial \mathcal{G}(x,-d ; l, \zeta)}{\partial \xi}\right) \mathrm{d} \zeta \\
& \quad+\lim _{z \uparrow-d} \int_{0}^{l}\left(\left(\mathcal{D} \frac{\partial^{4}}{\partial \xi^{4}}-\mu+1\right) w(\xi) \frac{\partial \mathcal{G}(x, z ; \xi,-d)}{\partial \zeta}-K w(\xi) \mathcal{G}(x, z ; \xi,-d)\right) \mathrm{d} \xi .
\end{aligned}
$$

We take the limit in the last integral after we have carried out the spatial integrations analytically. This means that we keep the factor $2 \pi$ in the left hand side of the equation. The commonly used factor $\pi$ and principle value integral may be obtained by taking the limit first. However, it is more convenient to avoid the principle value integral in our approach. In the first integral on the 
right-hand side we insert for the Green function the series expansion (15) and for the potential function the expansions (19) and (20), while in the second integral we use (14) for the Green function and (18) for the deflection. In the first integral integration with respect to $\zeta$ and in the last integral the integration with respect to $\xi$ can be carried out. Next we close the remaining contour of integration in the complex $\gamma$-plane.

If we now equalize the coefficients of $\mathrm{e}^{\mathrm{i} \kappa_{n} x}$ and of $\mathrm{e}^{-\mathrm{i} \kappa_{n}(x-l)}$, we obtain the following 'dispersion' relation for $\kappa_{n}$, the $\kappa_{n}$ 's are the zero's of

$$
\left(\mathcal{D} \kappa^{4}-\mu\right) K \cosh \kappa d+\left(K^{2}-\kappa^{2}\left(\mathcal{D} \kappa^{4}-\mu+1\right)\right) \frac{\sinh \kappa d}{\kappa}=\left(\mathcal{D} \kappa^{4}-\mu+1\right) \frac{(K \cosh \kappa h-\kappa \sinh \kappa h)}{\cosh \kappa(-d+h)} .
$$

After some manipulations this relation can be rewritten in the form:

$$
\left(\left(\mathcal{D} \kappa^{4}-\mu-1\right) \kappa \tanh \kappa(h-d)-K\right)(K \sinh \kappa d-\kappa \cosh \kappa d)=0
$$

For $d=0$ the dispersion relation for the zero draft platform is recovered. It should be noticed that relation (22) is not exactly the same as the zero draft relation with $h$ replaced by $h-d$. Hence, we ignore the zeros of the second part, that occur for values of $K$ sufficiently large only.

\subsection{Semi infinite platform}

Let us first consider the half-plane problem. We introduce some slight physical damping to get rid of the contributions of the upper bound in the last integral in (21) and the second part of the first integral. The terms we obtain after closure of the contour in the last integral of (21) contain the exponential functions $\mathrm{e}^{\mathrm{i} k_{n} x}$. We take the coefficients of each exponential equal to zero. This leads to a set of $N$ algebraic equations for the coefficients $a_{n}$ and $\alpha_{n}$. For the the half-plane problem, we obtain for $i=0, \cdots, N-1$ :

$$
\begin{array}{r}
\sum_{n=0}^{N-1} \frac{\alpha_{n}}{\cosh k_{n} h} \mathcal{K}_{i, n}-\sum_{n=0}^{N+1} \frac{a_{n}}{\kappa_{n}-k_{i}}\left(\left(\mathcal{D} \kappa_{n}^{4}-\mu+1\right) \sinh k_{i}(h-d)-\frac{K}{k_{i}} \cosh k_{i}(h-d)\right)= \\
\delta_{i}^{0} \frac{h k_{0}^{2}-h K^{2}+K}{\left.\left(k_{0}^{2}-K^{2}\right) \cosh k_{0} h\right)}-\frac{\mathcal{K}_{i, 0}}{\cosh k_{0} h},
\end{array}
$$

where the coefficients $\mathcal{K}_{i, n}$ are defined as

$$
\begin{aligned}
2 \mathcal{K}_{i, n} & \left.=\frac{1}{k_{i}+k_{n}}\left[\sinh \left(k_{i}+k_{n}\right) h-\sinh \left(k_{i}+k_{n}\right)(h-d)\right)\right] \\
& +\frac{1}{k_{i}-k_{n}}\left[\sinh \left(k_{i}-k_{n}\right) h-\sinh \left(k_{i}-k_{n}\right)(h-d)\right] .
\end{aligned}
$$

This is a set of $N$ equations for $2 N+2$ unknown coefficients. We have two conditions at the edge of the plate, so we must still obtain $N$. At the vertical frontend of the platform (17) gives the relation

$$
\begin{gathered}
2 \pi \phi(0, z)=2 \pi \phi^{\mathrm{inc}}+\lim _{x \rightarrow 0} \int_{-d}^{0} \phi(0, \zeta) \frac{\partial \mathcal{G}(x, z ; 0, \zeta)}{\partial \xi} \mathrm{d} \zeta \\
-\mathrm{i} \omega \int_{0}^{\infty}\left(\frac{1}{K}\left(\mathcal{D} \frac{\partial^{4}}{\partial \xi^{4}}-\mu+1\right) w(\xi) \frac{\partial \mathcal{G}(0, z ; \xi,-d)}{\partial \zeta}-w(\xi) \mathcal{G}(0, z ; \xi,-d)\right) \mathrm{d} \xi .
\end{gathered}
$$

We insert the series expansions (18) and (19) in this equation and compare the coefficients of $\cosh k_{i}(z+h)$. For the Green function we use expression (15) in both integrals.

We obtain for $i=0, \cdots, N-1$ :

$$
\begin{aligned}
& \frac{h k_{i}^{2}-h K^{2}+K}{\left(k_{i}^{2}-K^{2}\right) \cosh k_{i} h} \alpha_{i}-\sum_{n=0}^{N-1} \frac{\alpha_{n}}{\cosh k_{n} h} \mathcal{K}_{i, n} \\
& -\sum_{n=0}^{N+1} \frac{a_{n}}{\kappa_{n}+k_{i}}\left(\left(\mathcal{D} \kappa_{n}^{4}-\mu+1\right) \sinh k_{i}(h-d)-\frac{K}{k_{i}} \cosh k_{i}(h-d)\right)=\frac{1}{\cosh k_{0} h} \mathcal{K}_{i .0}
\end{aligned}
$$


We give some result for the absolute value of the amplitude of the deflection for a semi-infinite platform with a draft of two meters and water depth of ten meter. In figure 1 results are shown for three values of the deep water wave length, $\lambda=2 \pi / K=150,90,30 \mathrm{~m}$ respectively. As expected the amplitude increases with increasing values of the wave length. In figure 2 we show for $\lambda=90 \mathrm{~m}$ and water depth of ten meter the absolute value of the amplitude for several values of the draft, $d=0,2,4,6 \mathrm{~m}$. In figure 3 we show the influence of water depth on de amplitude
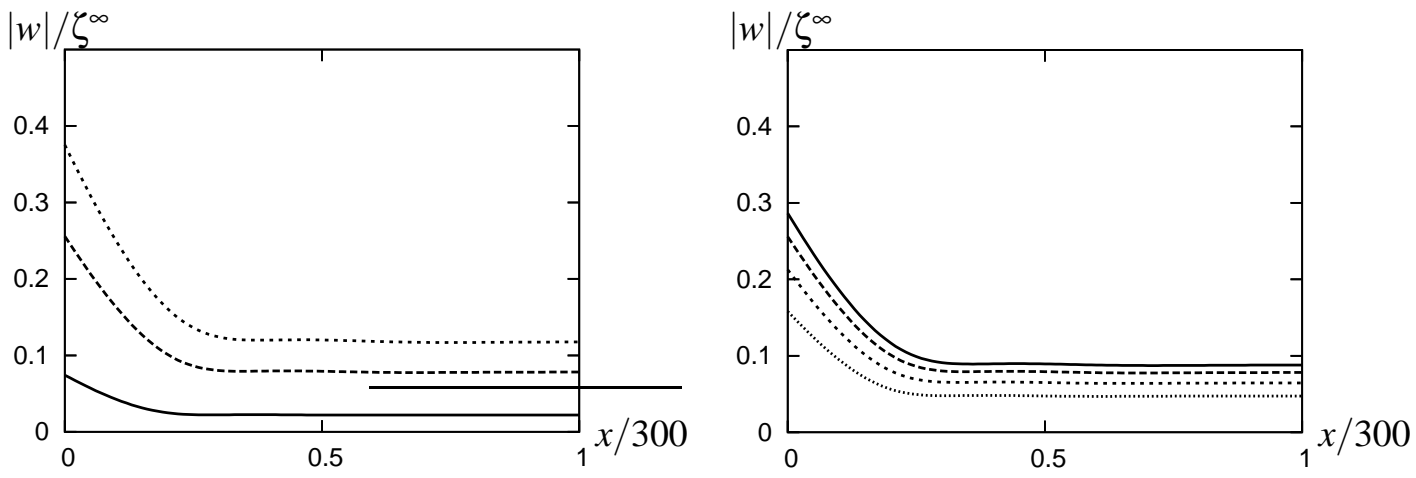

Figure 1: $\mathcal{D}=10^{7} \mathrm{~m}^{4}, d=2 \mathrm{~m}, h=10 \mathrm{~m}$ and $\lambda=150,90,30 \mathrm{~m}$ (top-down)

Figure 2: $\mathcal{D}=10^{7} \mathrm{~m}^{4}, d=0,2,4,6$ (topdown), $h=10 \mathrm{~m}$ and $\lambda=90 \mathrm{~m}$

of deflection. We have chosen $h=100,20,10 \mathrm{~m}, d=2 \mathrm{~m}$ and a fixed frequency with $\lambda=90 \mathrm{~m}$. The amplitude of the deflection increases for increasing water depth. To carry out computations for the larger values of water depth one must get rid of all hyperbolic sin and cosine functions in the formulation. This can be done by using standard formulas for these functions and by using the dispersion relation for the free surface water waves. By doing so one obtains very accurate results. In figure 4 we show the real part of the deflection for the same values of water depth, $d=5 \mathrm{~m}$ and fixed values of the wavelength $\lambda_{0}=2 \pi / k_{0}=100 \mathrm{~m}$. We also have computed the absolute value of the amplitude of the wave elevation in front of the platform. The result is shown in figure 5. It is clearly shown that the elevation of the wave and the platform are discontinuous at $x=0$. The amplitude of the reflected wave $\alpha_{0}=0.45657-0.43639$.
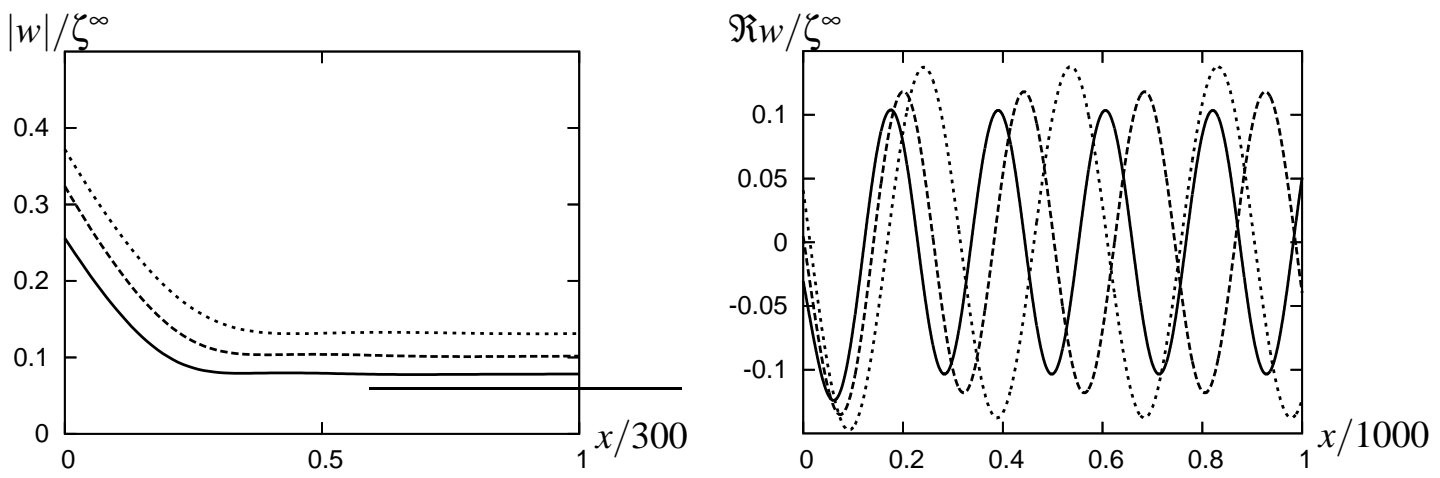

Figure 3: $\mathcal{D}=10^{7} \mathrm{~m}^{4}, d=2 \mathrm{~m}, h=$ $100,20,10 \mathrm{~m}$ (top-down) and $\lambda=90 \mathrm{~m}$
Figure 4: Real part of the deflection for $\mathcal{D}=$ $10^{7} \mathrm{~m}^{4}, d=5 \mathrm{~m}, h=100,20,10 \mathrm{~m}$ (topdown) $\mathrm{m}$ and $\lambda_{0}=100 \mathrm{~m}$

\subsection{Strip of finite length}

We follow the same procedure as for the semi-infinite case. The first step is to compare the coefficients of the exponential functions $\mathrm{e}^{ \pm \mathbf{i} k_{n} x}$ in (21). This leads to a set of $2 N$ algebraic equations for 


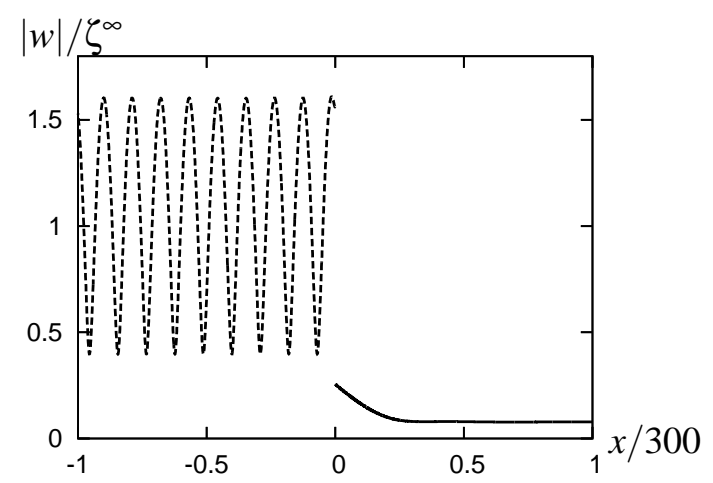

Figure 5: Amplitude of wave and deflection for $\mathcal{D}=10^{7} \mathrm{~m}^{4}, d=2 \mathrm{~m}, h=10 \mathrm{~m}$ and $\lambda=90 \mathrm{~m}$ the coefficients $a_{n}, b_{n}, \alpha_{n}$ and $\beta_{n}$.

$$
\begin{array}{r}
\sum_{n=0}^{N-1} \frac{\alpha_{n}}{\cosh k_{n} h} \mathcal{K}_{i, n}-\sum_{n=0}^{N+1} \frac{a_{n}}{\kappa_{n}-k_{i}}\left(\left(\mathcal{D} \kappa_{n}^{4}-\mu+1\right) \sinh k_{i}(h-d)-\frac{K}{k_{i}} \cosh k_{i}(h-d)\right) \\
+\sum_{n=0}^{N+1} \frac{b_{n}}{\kappa_{n}+k_{i}}\left(\left(\mathcal{D} \kappa_{n}^{4}-\mu+1\right) \sinh k_{i}(h-d)-\frac{K}{k_{i}} \cosh k_{i}(h-d)\right) \mathrm{e}^{\mathrm{i} \kappa_{n} l} \\
=\delta_{i}^{0} \frac{h k_{0}^{2}-h K^{2}+K}{\left.\left(k_{0}^{2}-K^{2}\right) \cosh k_{0} h\right)}-\frac{\mathcal{L}_{i, 0}}{\cosh k_{0} h}
\end{array}
$$

and

$$
\begin{aligned}
\sum_{n=0}^{N-1} \frac{\beta_{n}}{\cosh k_{n} h} \mathcal{K}_{i, n} & +\sum_{n=0}^{N+1} \frac{a_{n}}{\kappa_{n}+k_{i}}\left(\left(\mathcal{D} \kappa_{n}^{4}-\mu+1\right) \sinh k_{i}(h-d)-\frac{K}{k_{i}} \cosh k_{i}(h-d)\right) \mathrm{e}^{\mathrm{i} \kappa_{n} l} \\
& -\sum_{n=0}^{N+1} \frac{b_{n}}{\kappa_{n}+k_{i}}\left(\left(\mathcal{D} \kappa_{n}^{4}-\mu+1\right) \sinh k_{i}(h-d)-\frac{K}{k_{i}} \cosh k_{i}(h-d)\right)=0 .
\end{aligned}
$$

This is a set of $2 N$ equations for $4 N+4$ unknown coefficients. Next we consider the equations at $x=0$ and $x=l$ respectively. After integration with respect to the spatial variable one obtains a summation of $\cosh k_{i}(z+h)$ terms. By taking the coefficients of each $\cosh k_{i}(z+h)$ function equal to zero we obtain the following set of $2 N$ equations for the unknown expansion coefficients.

At $x=0$ we get

$$
\begin{aligned}
& \frac{h k_{i}^{2}-h K^{2}+K}{\left(k_{i}^{2}-K^{2}\right) \cosh k_{i} h} \alpha_{i}-\sum_{n=0}^{N-1} \frac{\alpha_{n}-\beta_{n} \mathrm{e}^{\mathrm{i} k_{i} l}}{\cosh k_{n} h} \mathcal{K}_{i, n} \\
& -\sum_{n=0}^{N+1} \frac{a_{n}}{\kappa_{n}+k_{i}}\left(\left(\mathcal{D} \kappa_{n}^{4}-\mu+1\right) \sinh k_{i}(h-d)-\frac{K}{k_{i}} \cosh k_{i}(h-d)\right)\left(1-\mathrm{e}^{\mathrm{i}\left(\kappa_{n}+k_{i}\right) l}\right) \\
& +\sum_{n=0}^{N+1} \frac{b_{n}}{\kappa_{n}-k_{i}}\left(\left(\mathcal{D} \kappa_{n}^{4}-\mu+1\right) \sinh k_{i}(h-d)-\frac{K}{k_{i}} \cosh k_{i}(h-d)\right)\left(\mathrm{e}^{\mathrm{i} \kappa_{n} l}-\mathrm{e}^{\mathrm{i} k_{i} l}\right) \\
& =\frac{1}{\cosh k_{0} h} \mathcal{K}_{i .0},
\end{aligned}
$$

and at $x=l$ we get

$$
\begin{gathered}
\frac{h k_{i}^{2}-h K^{2}+K}{\left(k_{i}^{2}-K^{2}\right) \cosh k_{i} h} \beta_{i}+\sum_{n=0}^{N-1} \frac{\alpha_{n} \mathrm{e}^{\mathrm{i} k_{i} l}-\beta_{n}}{\cosh k_{n} h} \mathcal{K}_{i, n} \\
+\sum_{n=0}^{N+1} \frac{a_{n}}{\kappa_{n}-k_{i}}\left(\left(\mathcal{D} \kappa_{n}^{4}-\mu+1\right) \sinh k_{i}(h-d)-\frac{K}{k_{i}} \cosh k_{i}(h-d)\right)\left(\mathrm{e}^{\mathrm{i} \kappa_{n} l}-\mathrm{e}^{\mathrm{i} k_{i} l}\right) \\
-\sum_{n=0}^{N+1} \frac{b_{n}}{\kappa_{n}+k_{i}}\left(\left(\mathcal{D} \kappa_{n}^{4}-\mu+1\right) \sinh k_{i}(h-d)-\frac{K}{k_{i}} \cosh k_{i}(h-d)\right)\left(1-\mathrm{e}^{\mathrm{i}\left(\kappa_{n}+k_{i}\right) l}\right) \\
=\frac{h k_{0}^{2}-h K^{2}+K}{\left(k_{0}^{2}-K^{2}\right) \cosh k_{0} h} \mathrm{e}^{\mathrm{i} k_{0} l}-\frac{1}{\cosh k_{0} h} \mathcal{K}_{i .0}
\end{gathered}
$$


Together with the four relations at the end of the strip we have $4 N+4$ linear algebraic equations for the $4 N+4$ unknown coefficients.

The set of equations as is written here is not very suitable for numerical computations directly. Especially for large values of water-depth the arguments of the hyperbolic sine and cosine functions becomes rather large. So one is subtracting very large values in the computation of the coefficients. To obtain high numerical accuracy one must get rid of these functions. This can be done by using the dispersion relation for the water region. In Appendix B a more suitable set of equations is given.

We show some computational results for a two-dimensional platform of width $300 \mathrm{~m}$. In all cases we take a fixed values for the flexural rigidity $\mathcal{D}=10^{7} \mathrm{~m}^{4}$, the width of the strip $l=300 \mathrm{~m}$ and the water depth $h=10 \mathrm{~m}$. In figure 6 and 7 we show for $d=0$ and for $d=2 \mathrm{~m}$ the variation of the amplitude of deflection with respect to the wave length.
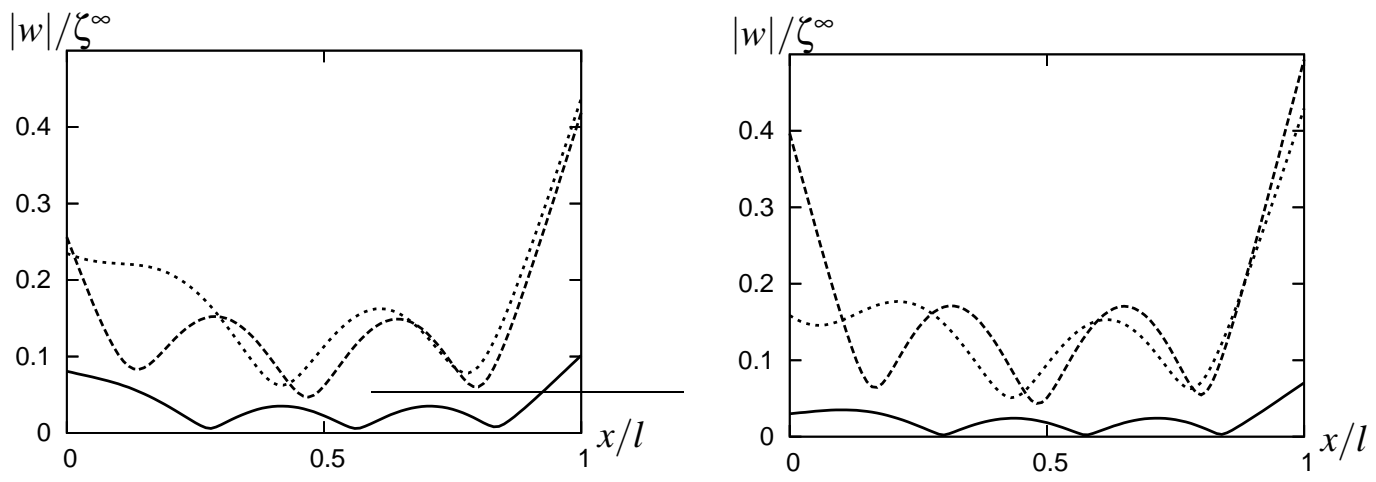

Figure 6: $\quad \mathcal{D}=10^{7} \mathrm{~m}^{4}, \quad l=300$ $\mathrm{m}, \quad d=0 \mathrm{~m}, \quad h=10 \mathrm{~m} \quad$ and $\lambda / l=0.5, \cdots, 0.3,--, 0.1,-$

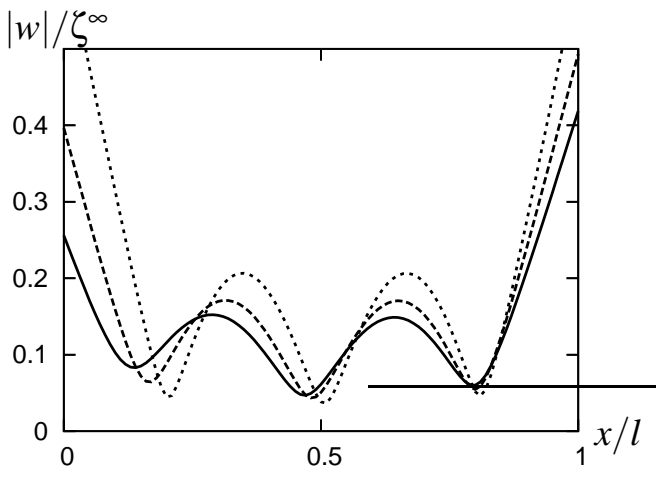

Figure 7: $\mathcal{D}=10^{7} \mathrm{~m}^{4}, l=300 \mathrm{~m}, d=2 \mathrm{~m}$, $h=10 \mathrm{~m}$ and $\lambda / l=0.5,0.3,0.1$

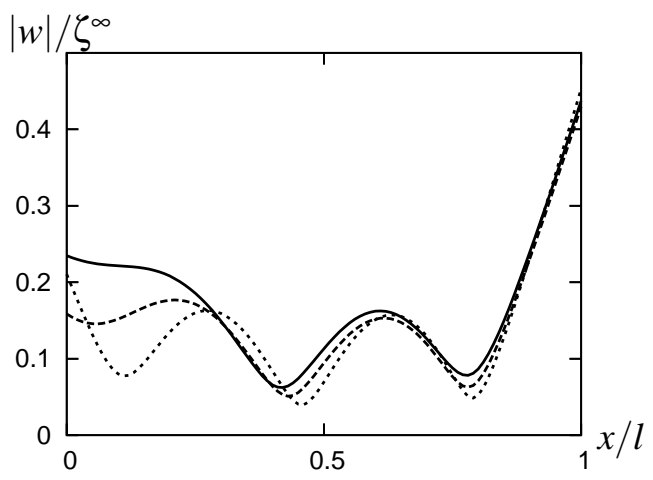

Figure 8: $\mathcal{D}=10^{7} \mathrm{~m}^{4}, l=300 \mathrm{~m}, d=0,-$,

Figure 9: $\mathcal{D}=10^{7} \mathrm{~m}^{4}, l=300 \mathrm{~m}, d=0,-$, $2,--, 4, \cdots \mathrm{m}, h=10 \mathrm{~m}$ and $\lambda / l=0.3$ $2,--, 4, \cdots \mathrm{m}, h=10 \mathrm{~m}$ and $\lambda / l=0.5$

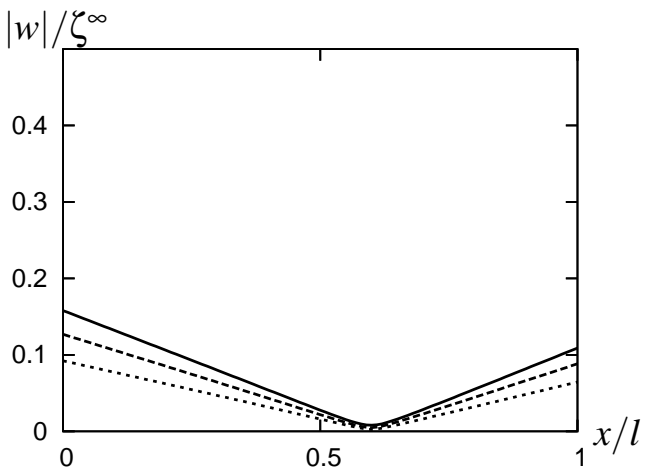

Figure 10: $\mathcal{D}=10^{10} \mathrm{~m}^{4}, l=300 \mathrm{~m}, d=0,2,4 \mathrm{~m}, h=10 \mathrm{~m}$ and $\lambda / l=0.5$ 


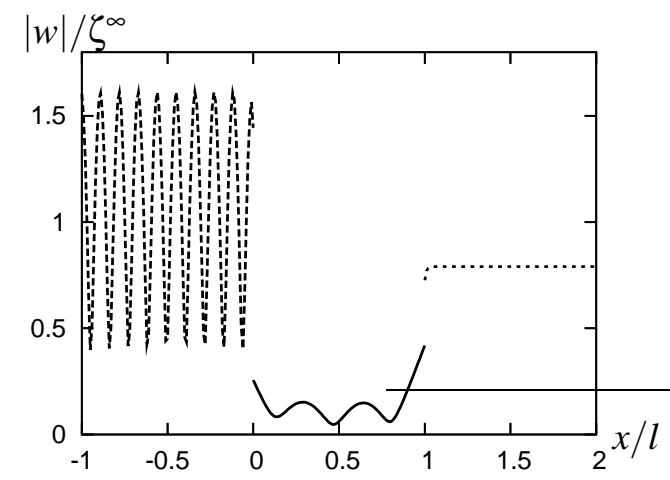

Figure 11: $\mathcal{D}=10^{7} \mathrm{~m}^{4}, l=300 \mathrm{~m}, d=0$ $\mathrm{m}, h=10 \mathrm{~m}$ and $\lambda / l=0.3$

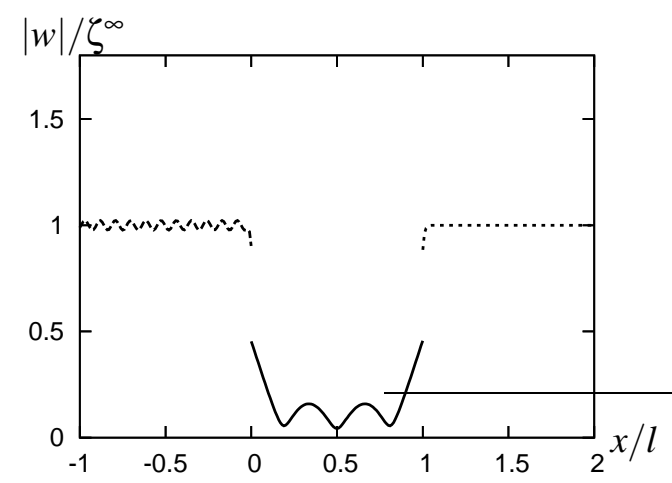

Figure 13: $\mathcal{D}=10^{7} \mathrm{~m}^{4}, l=300 \mathrm{~m}, d=0$ $\mathrm{m}, h=10 \mathrm{~m}$ and $\lambda / l=0.215$

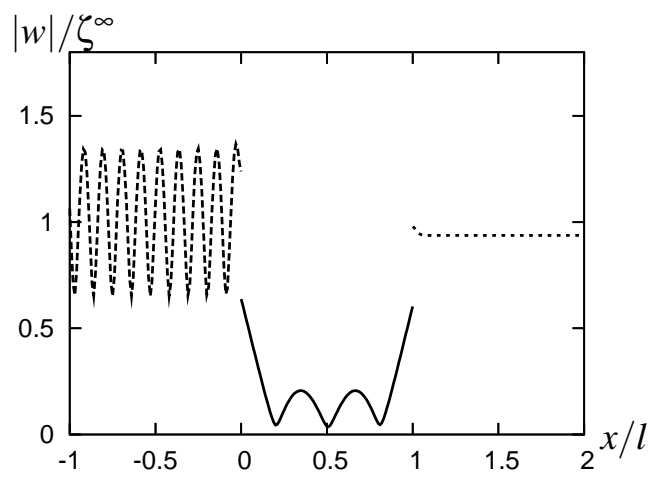

Figure 12: $\mathcal{D}=10^{7} \mathrm{~m}^{4}, l=300 \mathrm{~m}, d=4$ $\mathrm{m}, h=10 \mathrm{~m}$ and $\lambda / l=0.3$

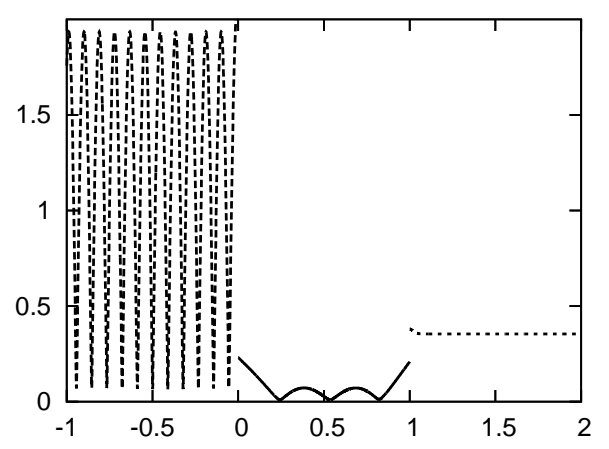

Figure 14: $\mathcal{D}=10^{7} \mathrm{~m}^{4}, l=300 \mathrm{~m}, d=4$ $\mathrm{m}, h=10 \mathrm{~m}$ and $\lambda / l=0.215$

In figure 8 and 9 the dependence on the draft for fixed values of the wave length is shown. The results of the first case show an increase of the deflection for increasing values of the draft. It will be shown later that this is due to a shift in the reflection curve. In figure 10 a result is shown for a larger value of the flexural rigidity $\mathcal{D}=10^{10} \mathrm{~m}^{4}$ and wave length $\lambda / l=0.5$. This case is comparable with the interaction of free-surface waves with a rigid body. One clearly observes that the motion of the dock consists of a heave and pitch motion only.

In figure 11 and 13 we show for two values of the wave length the absolute value of the amplitude of the water surface in front and behind the strip, together with the amplitude of the plate deflection for the zero draft case. The second case is near the zero reflection situation.

In the four meter draft case, see figure 12 and 14 , we see that $\lambda / l=0.215$, or in terms of the actual wave length $\lambda_{0} / l=0.178$, is close to total reflection. This is in contrast with the zero draft case in figure 13, due to the shift in the transmission-reflection curves. For the same reason the absolute value of the deflection increases if the draft increases in figure 8 in contrast with the result in figure 9.

The reflection and transmission coefficients for a strip of $300 \mathrm{~m}$ and depth $10 \mathrm{~m}$ are shown in figure 15, for zero draft and in 16 for a draft of two meter. If we define $R=\alpha_{0}$ and $T=\beta_{0}$, notice no exponential function, we find that in all cases the relations $|T|^{2}+|R|^{2}=1$ and $T \bar{R}+\bar{T} R=0$, see for instance Mei et al [1] or for a derivation Roseau [8], are fulfilled for at least 10 decimals. The coefficients are presented as a function of the actual wave length, $\lambda_{0} / l=2 \pi / k_{0} l$. In the figures 17 and 18 these coefficients are given for a water depth of 100 meter. In all cases the coefficient of flexural rigidity equals $\mathcal{D}=10^{7} \mathrm{~m}^{4}$. Figures 18,19 and 20 show the results for different sizes of the strip. In figure 21 and 22 the result is shown for a strip of width $l=100 \mathrm{~m}$ and draft $d=8 \mathrm{~m}$. It is clearly observed that for the short waves total reflection takes place . 

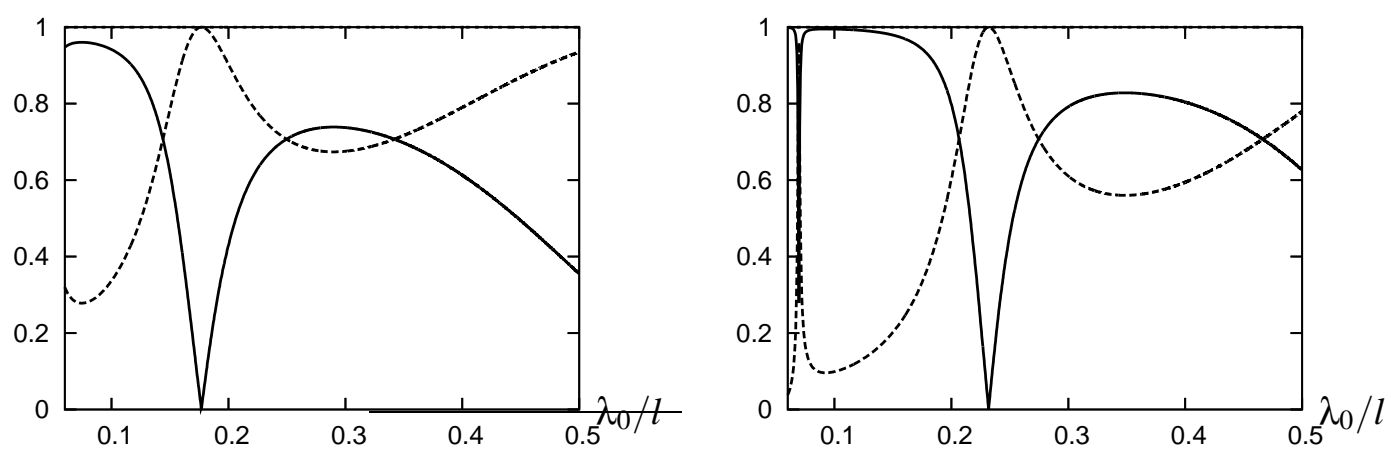

Figure 15: -- Reflection and -- transmission coefficients for $h=10 \mathrm{~m}, d=0 \mathrm{~m}$ and $l=300 \mathrm{~m}$

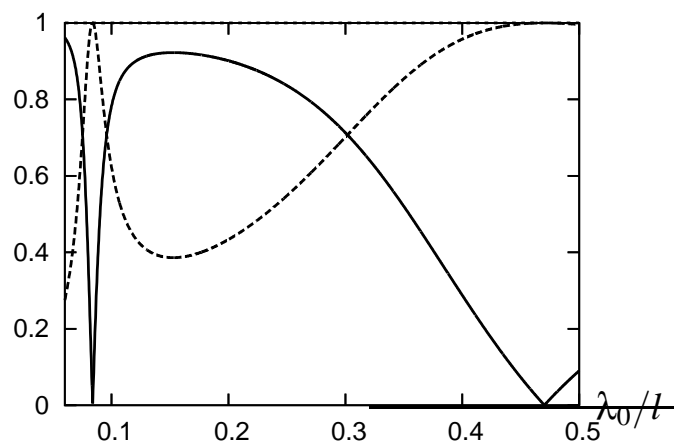

Figure 16: Reflection and transmission coefficients for $h=10 \mathrm{~m}, d=4 \mathrm{~m}$ and $l=$ $300 \mathrm{~m}$

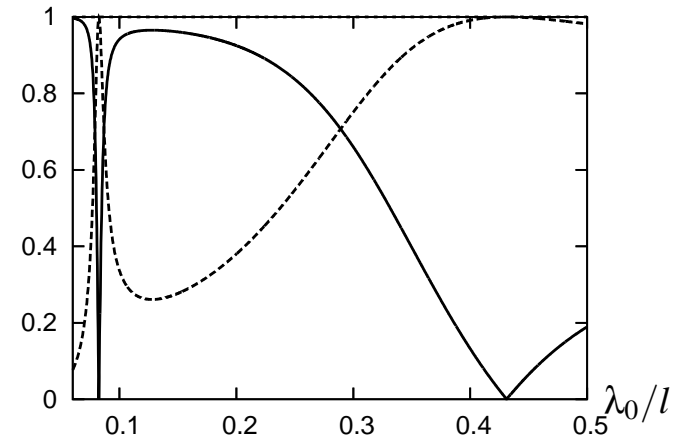

Figure 17: Reflection and transmission coefficients for $h=100 \mathrm{~m}, d=0 \mathrm{~m}$ and $l=$ $300 \mathrm{~m}$

Figure 18: Reflection and transmission coefficients for $h=100 \mathrm{~m}, d=2 \mathrm{~m}$, and $l=$ $300 \mathrm{~m}$

\section{A The Green's function}

Here we derive the two-dimensional version of the function of Green $\mathcal{G}(x, z ; \xi, \zeta)$ as used in this paper. This 'source' function is a solution of

$$
\mathcal{G}_{x x}+\mathcal{G}_{z z}=2 \pi \delta(x-\xi, z-\zeta)
$$

with boundary conditions:

$$
\begin{gathered}
K \mathcal{G}-\mathcal{G}_{z}=0 \text { at } z=0 \\
\mathcal{G}_{z}=0 \text { at } z=-h .
\end{gathered}
$$

We introduce the Fourier transform of $\mathcal{G}$

$$
\tilde{\mathcal{G}}(z ; \zeta)=\frac{1}{2 \pi} \int_{-\infty}^{\infty} \mathcal{G}(x, z ; \xi, \zeta) \mathrm{e}^{-\mathrm{i} \gamma x} \mathrm{~d} x .
$$

This transformed Green's function satisfies the conditions:

$$
\begin{aligned}
\tilde{\mathcal{G}}_{z z}-\gamma^{2} \tilde{\mathcal{G}} & =0 & & \text { for } z \neq \zeta \\
K \tilde{\mathcal{G}}-\tilde{\mathcal{G}} & =0 & & \text { at } z=0 \\
\tilde{\mathcal{G}}_{z} & =0 & & \text { at } z=-h \\
\lim _{\varepsilon \rightarrow 0}(\tilde{\mathcal{G}}(\zeta+\varepsilon ; \zeta)-\tilde{\mathcal{G}}(\zeta-\varepsilon ; \zeta)) & =0 & & \\
\lim _{\varepsilon \rightarrow 0}\left(\tilde{\mathcal{G}}_{z}(\zeta+\varepsilon ; \zeta)-\tilde{\mathcal{G}}_{z}(\zeta-\varepsilon ; \zeta)\right) & =\mathrm{e}^{-\mathrm{i} \gamma \xi} . & &
\end{aligned}
$$

The solution of this equations is

$$
\tilde{\mathcal{G}}(z ; \zeta)=\frac{1}{\gamma} \frac{K \sinh \gamma z+\gamma \cosh \gamma z}{K \cosh \gamma h-\gamma \sinh \gamma h} \cosh \gamma(\zeta+h) \mathrm{e}^{-\mathrm{i} \gamma \xi} \text { for } z>\zeta
$$



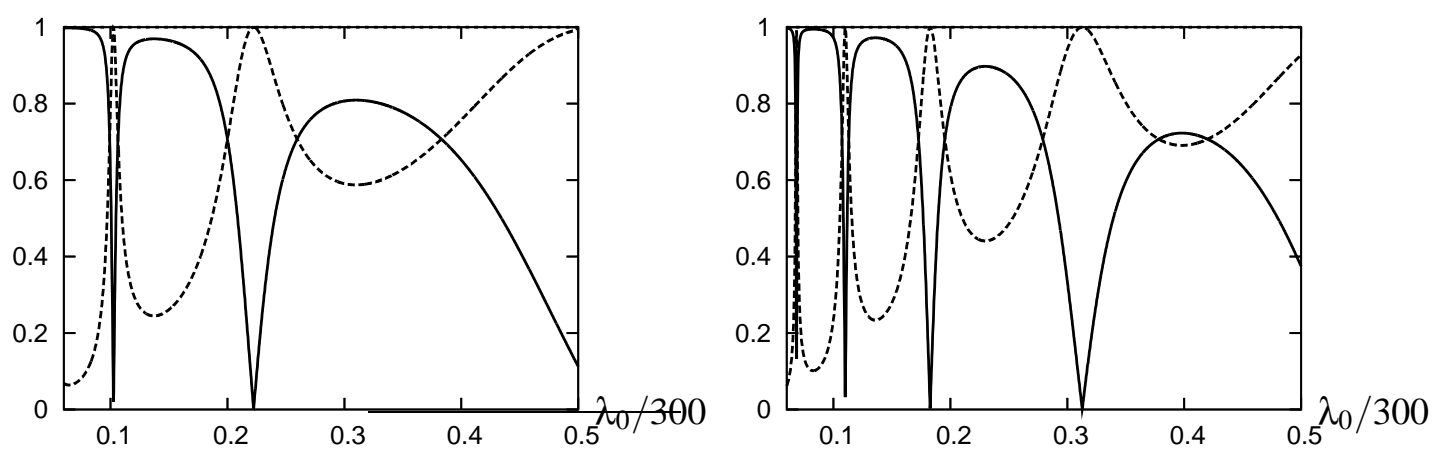

Figure 19: Reflection and transmission coefficients for $h=100 \mathrm{~m}, d=2 \mathrm{~m}$, and $l=$ $650 \mathrm{~m}$

Figure 20: Reflection and transmission coefficients for $h=100 \mathrm{~m}, d=2 \mathrm{~m}$ and $l=$ $1000 \mathrm{~m}$
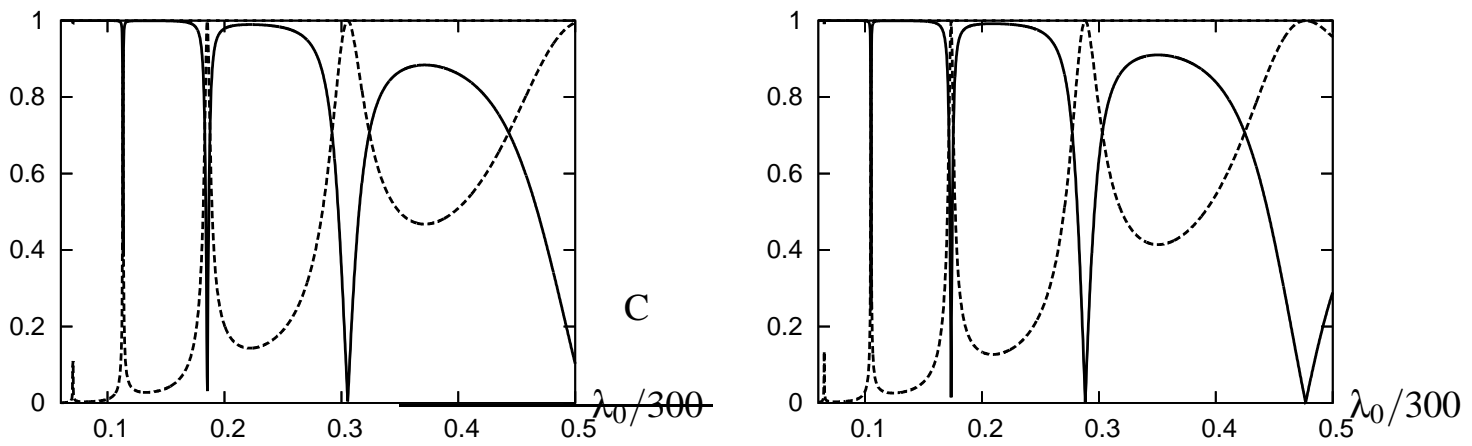

Figure 21: Reflection and transmission coefficients for $h=100 \mathrm{~m}, d=8 \mathrm{~m}$, and $l=$ $1000 \mathrm{~m}$

Figure 22: Reflection and transmission coefficients for $h=500 \mathrm{~m}, d=8 \mathrm{~m}$ and $l=$ $1000 \mathrm{~m}$

$$
\tilde{\mathcal{G}}(z ; \zeta)=\frac{1}{\gamma} \frac{K \sinh \gamma \zeta+\gamma \cosh \gamma \zeta}{K \cosh \gamma h-\gamma \sinh \gamma h} \cosh \gamma(z+h) \mathrm{e}^{-\mathrm{i} \gamma \xi} \text { for } z<\zeta .
$$

Then we transformed back to the $x$-variable. This results in (13) and (14. The contour of integration passes above or underneath the singularities on the real axis. The choice of this contour is determined by the radiation condition. For $x>\xi$ the waves travel in the positive $x$-direction, while for $x<\xi$ the waves travel in the negative $x$-direction. Therefore the contour passes the negative real pole above and the positive real pole below. Closure of the contour in the complex $\gamma$-plane leads to (15).

\section{B Simplification of the set of algebraic equations}

To obtain accurate solutions of the set of equations (26-28) one must get rid of the terms that consist of subtraction of large numbers. To achieve this goal we use the dispersion relation (10), $\gamma \tanh (\gamma h)=K$ Making use of the relation

$$
\cosh (\gamma h)^{2}-\sinh (\gamma h)^{2}=1
$$

one obtains for the zeros $\gamma=k_{i}$ for $i=0,1, \cdots \ldots$

$$
\cosh \left(k_{i} h\right)=\frac{(-1)^{i} k_{i}}{\sqrt{k_{i}^{2}-K^{2}}} \text { and } \sinh \left(k_{i} h\right)=\frac{(-1)^{i} K}{\sqrt{k_{i}^{2}-K^{2}}} .
$$

We also use

$$
\begin{aligned}
& \cosh \gamma(h-d)=\cosh \gamma h \cosh \gamma d-\sinh \gamma h \sinh \gamma d \\
& \sinh \gamma(h-d)=\sinh \gamma h \cosh \gamma d-\cosh \gamma h \sinh \gamma d .
\end{aligned}
$$


One can see that for large values depth $k_{0}$ is very close to $K$ the accuracy is improved if one divides out the large term analytically. The results in 21 can not be obtained without this simplification.

\section{References}

[1] C.C. Mei and J.L. Black, Scattering of surface waves by rectangular obstacles in waters of finite depth. J. of Fluid Mechanics 38 (1969) 499-511.

[2] A.J. Hermans, Interaction of free-surface waves with a floating dock, J. of Engineering Mathematics 45 (2003) 39-53.

[3] J.V. Wehausen, and E.V. Laitone, Surface waves. Encyclopedia of Physics 9, Springer-Verlag, Berlin, (1960) 446-814, also http://www.coe.berkeley.edu/SurfaceWaves/.

[4] M.H. Meylan and V.A. Squire, The response of a thick flexible raft to ocean waves. International Journal of Offshore and Polar Engineering 5 (1995), 198-203.

[5] A.J. Hermans, A Boundary Element Method for the Interaction of Free-Surface Waves with a Very Large Floating Flexible Platform. J. of Fluids and Structures 14 (2000) 943-956.

[6] A.J. Hermans, The ray method for the deflection of a floating flexible platform in short waves J. of Fluids and Structures 17 (2003) 593-602.

[7] Hyuck Chung, C. M. Linton,,Interaction between water waves and elastic plates : Using the residue calculus technique, Proceedings of the 18th IWWWFB edited by A.H. Clément and P. Ferrant, Ecole Centrale de Nantes, France (2003).

[8] M. Roseau, Asymptotic wave theory, North-Holland Publishing Company, (1976). 